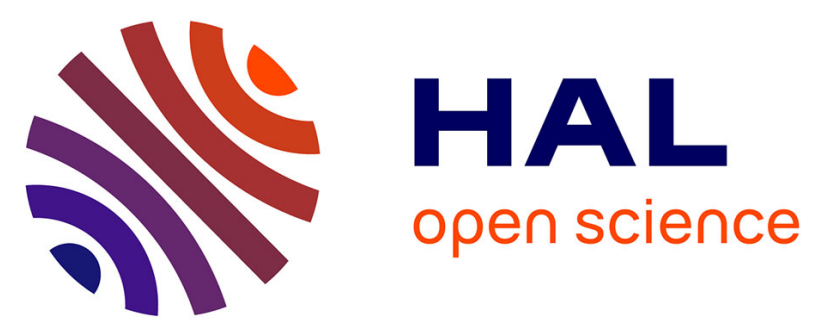

\title{
Message-Passing Algorithm for Sum-rate Maximization in Multi-antenna Underlay D2D Communications
}

\author{
Berna Ozbek, Mylene Pischella, Didier Le Ruyet
}

\section{To cite this version:}

Berna Ozbek, Mylene Pischella, Didier Le Ruyet. Message-Passing Algorithm for Sumrate Maximization in Multi-antenna Underlay D2D Communications. 2018 15th International Symposium on Wireless Communication Systems (ISWCS), Aug 2018, Lisbonne, Portugal. 10.1109/ISWCS.2018.8491058 . hal-02446288

\section{HAL Id: hal-02446288 https://hal-cnam.archives-ouvertes.fr/hal-02446288}

Submitted on 19 Mar 2020

HAL is a multi-disciplinary open access archive for the deposit and dissemination of scientific research documents, whether they are published or not. The documents may come from teaching and research institutions in France or abroad, or from public or private research centers.
L'archive ouverte pluridisciplinaire HAL, est destinée au dépôt et à la diffusion de documents scientifiques de niveau recherche, publiés ou non, émanant des établissements d'enseignement et de recherche français ou étrangers, des laboratoires publics ou privés. 


\title{
Message-Passing Algorithm for Sum-rate Maximization in Multi-antenna Underlay D2D Communications
}

\author{
Berna Özbek ${ }^{1}$, Mylene Pischella ${ }^{2}$, Didier Le Ruyet ${ }^{2}$ \\ ${ }^{1}$ Izmir Institute of Technology, Dept. of Electrical and Electronics Eng., Izmir, Turkey. \\ ${ }^{2}$ CEDRIC/LAETITIA, CNAM, Paris, France. \\ e-mail:\{bernaozbek@iyte.edu.tr\},\{mylene.pischella,leruyet@cnam.fr\}
}

\begin{abstract}
In this paper, we propose a resource allocation of device-to-device (D2D) communications underlaying multiantenna cellular systems by employing message-passing algorithm. It is possible to construct the factor graph of the D2D pairs by considering the interference power at the base station and among the D2D pairs. The goal is to avoid interfering the cellular transmission while increasing the sum data rate of D2D pairs. We show that the proposed resource allocation based on message passing algorithm allow us to almost reach the performance of the exhaustive search and outperforms the random allocation. Since the associated factor graph is not fully connected, the complexity of the algorithm is significantly reduced compared to the exhaustive search.
\end{abstract}

\section{INTRODUCTION}

The need for higher data rate is exponentially increasing due to very high throughput services such as high quality video streaming, mobile applications and augmented reality. The need for high throughput implies an efficient allocation of the given resources. Since users often want to share data with others in their close vicinity, direct communications between the users or device-to-device (D2D) communications have recently received a significant interest. It is known that D2D communications [1] can be used in a cellular communication system to increase the data rate where the goal is to maximize D2D data rate without affecting the cellular data rate. Different optimization objectives can be considered such as maximizing the sum-rate, the overall energy efficiency, the fairness between the different users, etc. These problems can be solved using graph-coloring, bipartite matching or iterative methods [2] [3].

In this paper, our aim is to optimize the resource and power allocation of D2D pairs that are underlaid in uplink cellular multiantenna communications through optimal Signal to Interference plus Noise (SINR) ratios determination. The optimization objective is to maximize the sum-rate of D2D pairs and cellular communications in a given Resource Block (RB), given that cellular users are allocated prior to D2D pairs and under a maximum interference threshold per D2D pair at the Base Station (BS). Each D2D pair may choose several target SINR ratios, or be inactive. The allocation decision is obtained in a distributed way by performing the min- sum message-passing algorithm (MPA) over the factor graph describing the interference power between the different links.

Message-passing algorithms, whose sum-product variant is also called belief propagation, have been successively used in communication networks in different contexts, in order to solve various problems such as the decoding of low-density parity check (LDPC) codes, turbo codes or turbo equalization [4] with a lower complexity than decoding with maximumlikelihood criterion. The MPA converges to the maximumlikelihood optimal solution if the factor graph on which the MPA is perform is cycle-free. The factor graph is a bipartite graph that represents how the multivariate functions can be factorized. The MPA has also been used to perform resource allocation, with the min-sum variant, which is equivalent to the max-product MPA, in [5]-[8].

The rest of the paper is organized as follows. In Section II, we introduce the considered multi-antenna underlay D2D communications scenario and the associated system model based on a factor graph. In Section III, the optimization problem is defined and the proposed MPA for resource allocation is described. In Section IV, the simulation results are presented to demonstrate the interest of the proposed algorithm.

\section{SYSTEM MODEL}

In this work, we consider the uplink cellular communications underlaying D2D transmissions. This choice allows a better management of the interference generated by the D2D transmitters to the BS compared to downlink case. We focus on one RB of bandwidth $B_{c}$. The extension to Orthogonal Frequency Division Multiple Access (OFDMA) will be subject to the further studies. We consider a single cell case with a BS station equipped with $N_{r}$ antennas, $K_{c}$ single antenna cellular users and $K$ D2D pairs.

We define $\mathbf{H}_{k, 0}$ as the $1 \times N_{r}$ channel vector between the $k$ D2D transmitter and the $\mathrm{BS}, \mathbf{H}_{k_{c}, 0}^{\prime}$ as the $1 \times N_{r}$ channel vector between the $k_{c}$ cellular user and the BS. $g_{j, k}$ is the channel gain from D2D transmitter $j$ to D2D receiver $k$ and $g_{k_{c}, k}^{\prime}$ is the channel gain between the cellular user $k_{c}$ and D2D receiver $k$.

Let $s_{k}$ be the target SINR at D2D receiver $k$. It belongs to subset $\mathcal{S}=\left\{\mathrm{SINR}^{0}, \mathrm{SINR}^{1}, \ldots \mathrm{SINR}^{T-1}\right\}$, where $\operatorname{SINR}^{t}>$ 
$\operatorname{SINR}^{t-1}$ for $t \in\{1, \ldots, T-1\}, T$ is the number of allowed target SINR values. We have fixed $\operatorname{SINR}^{0}=0$ so that when SINR $^{0}$ is chosen by D2D pair $k$, it will be shut down. The possible values of the transmit power of D2D transmitter $k$ are, for any $t \in\{1, \ldots, T-1\}$ :

$$
P_{k}^{t}=\min \left\{P_{\max }, \frac{\mathrm{SINR}^{t} \times\left(N_{0} B_{c}+\widehat{I_{k}}\right)}{g_{k, k}}\right\}
$$

where $\widehat{I}_{k}$ is the estimated interference level at D2D receiver $k, N_{0}$ is the noise power spectral density and $P_{\max }$ is the maximum transmitted power allowed. The optimization objective is to determine, for each D2D pair, the optimal value of $s_{k} \in \mathcal{S}$, and subsequently the optimal transmit power value $P_{k}^{t}$.

At the BS, we perform Maximum Ratio Combining (MRC) in order to maximize the received power from the cellular user. First, the active cellular user having the highest channel gain after post-coding at the BS is selected among $K_{c}$ cellular users:

$$
k_{c}^{*}=\arg \max _{k_{c}=1, \ldots, K_{c}}\left|\mathbf{H}_{k_{c}, 0}^{\prime} \mathbf{G}_{k_{c}, 0}^{H}\right|^{2}
$$

where $\mathbf{G}_{k_{c}, 0}$ is the post-coding vector which is the normalized channel vector defined by,

$$
\mathbf{G}_{k_{c}, 0}=\frac{\mathbf{H}_{k_{c}, 0}^{\prime}}{\left\|\mathbf{H}_{k_{c}, 0}^{\prime}\right\|} ; \quad \forall k_{c} \in\left\{1, \ldots, K_{c}\right\}
$$

The cellular user allocated on the RB is indexed by 0 . Then, the channel gain between the selected cellular user and the BS after applying post-coding is given by,

$$
g_{0,0} \triangleq\left|\mathbf{H}_{k_{c}^{*}, 0}^{\prime} \mathbf{G}_{k_{c}^{*}, 0}^{H}\right|^{2}
$$

The cellular transmit power of the selected cellular user can be calculated with the objective to reach a fixed target SINR, $\mathrm{SINR}_{c}$ as follows:

$$
P_{0}=\min \left\{P_{\text {max }}, \frac{\operatorname{SINR}_{c} \times\left(N_{0} B_{c}+\widehat{I_{0}}\right)}{g_{0,0}}\right\}
$$

where $\widehat{I}_{0}$ is the estimated interference level at the BS.

In order to reduce the complexity of the MPA, some interference is neglected when building the factor graph. The factor graph per $\mathrm{RB}$ is composed of $K$ variables nodes (one per D2D transmitter) and $K+1$ factor nodes (one per D2D receiver and the $\mathrm{BS}$ ). It is built for each network state as follows: first, variable node $j \in\{1, \ldots, K\}$ is always connected to factor node $j$. Then, factor node $k \in\{1, \ldots, K\}$ is connected to variable node $j \in\{1, \ldots, K\}$, with $k \neq j$, if the estimated interference generated by D2D transmitter $j$ on D2D receiver $k$ is larger than a given threshold $I_{t h}$. The estimated interference is the worst-case interference computed when the transmit power of D2D transmitter $j$ is at its maximum value corresponding to $\mathrm{SINR}^{T-1}$. The connectivity constraint consequently is:

$$
g_{j, k} P_{j}^{T-1}>I_{t h}
$$

Similarly, the BS factor node 0 is connected to variable node $k \in\{1, \ldots, K\}$ if the worst-case estimated interference generated by D2D transmitter $k$ using $\operatorname{SINR}^{T-1}$ and postcoding at the BS is higher than a given threshold which is determined proportional to $N_{r}$ and $I_{t h}$ as:

$$
g_{k, 0} P_{k}^{T-1}>N_{r} I_{t h}
$$

where $g_{k, 0}$ is the channel gain between the $k$ th D2D transmitter and the BS after applying post-coding. It can be determined as follows:

$$
g_{k, 0}=\left|\mathbf{H}_{k, 0} \mathbf{G}_{k_{c}^{*}, 0}^{H}\right|^{2}
$$

The factor graph is not fully connected, except in very dense scenario. The complexity of MPA is in $\mathcal{O}\left(T^{d}\right)$, where $d$ is the maximum degree of factor nodes, i.e., the maximum number of interferers whose influence is large enough at the BS or the maximum number of interferers plus one at the $\mathrm{D} 2 \mathrm{D}$ receivers.

The data rate of each $\mathrm{D} 2 \mathrm{D}$ pair is given by,

$$
C_{k}=\log _{2}\left(1+\frac{P_{k}^{t} g_{k, k}}{N_{0} B_{c}+I_{k}+I_{0, k}}\right)
$$

where $I_{k}$ is the total eceived interference at the D2D receiver $k$ which is generated by all D2D transmitters except the D2D transmitter of interest $k$. This interference is determined as follows:

$$
I_{k}=\sum_{\substack{j=1 \\ j \neq k}}^{K} P_{j}^{t} g_{j, k}
$$

$I_{0, k}$ is the received interference at the $\mathrm{D} 2 \mathrm{D}$ receiver $k$ which generated by the selected cellular user. This interference is calculated by,

$$
I_{0, k} \triangleq P_{0} g_{0, k}^{\prime}
$$

The selected cellular data rate is determined by,

$$
C_{0}=\log _{2}\left(1+\frac{P_{0} g_{0,0}}{N_{0} B_{c}+I_{0}}\right)
$$

where $I_{0}$ is the received interference from all D2D transmitters at the BS and is given by,

$$
I_{0}=\sum_{k=1}^{K} I_{k, 0}
$$

where $I_{k, 0}$ is the total interference generated by the D2D transmitter $k$ after postcoding at the BS. This interference is determined as,

$$
I_{k, 0} \triangleq P_{k}^{t} g_{k, 0}
$$

The estimated interference at the BS and the D2D receiver $k$ are determined by considering the worst case scenario in which all D2D pairs are active as in the following:

$$
\begin{aligned}
& \widehat{I_{0}}=N_{r} K I_{t h} \\
& \widehat{I_{k}}=K I_{t h}
\end{aligned}
$$




\section{Proposed ALGORITHM}

\section{A. Optimization problem}

In this work, the optimization objective is to maximize the sum rate of D2D pairs and cellular communications per RB, given that cellular users are allocated prior to the D2D pairs and under a maximum interference threshold per D2D pair at the BS. The optimization variables are the D2D target SINR values, $\mathbf{S}=\left\{s_{1}, \ldots, s_{K}\right\}$. These variables are preferred to the transmit powers in order to ensure some fairness between D2D pairs. By adjusting the SINR values, the data rates are also adjusted, which means that any D2D pair should achieve one of the $T$ possible data rates corresponding to the $T$ target SINR.

The sum rate maximization problem can be written as follows:

$$
\begin{aligned}
& \max _{\mathbf{S} \in \mathcal{S}^{K}} C_{0}(\mathbf{S})+\sum_{k=1}^{K} C_{k}(\mathbf{S}) \\
& \text { s.t. } I_{k, 0} \leq N_{r} I_{\max } \forall k \in\{1, \ldots, K\}
\end{aligned}
$$

where $I_{\max }$ is the maximum allowed interference level per D2D transmitter at the BS. $C_{k}(\mathbf{S})$ is the achievable rate at D2D pair $k$, given that power values are computed using the target SINR set $\mathbf{S}$.

The data rate at $\mathrm{D} 2 \mathrm{D}$ receiver $k, C_{k}$, depends on set $\mathbf{S}$. Since some interference is neglected, as previously explained, $C_{k}$ can be written as a function of a subset of D2D SINR set, $\mathbf{S}_{\rightarrow k}$. It only contains the SINR of D2D pairs that are taken into account in the factor-graph. The set of indices kept in $\mathbf{S}_{\rightarrow k}$ with respect to $\mathbf{S}$ is the set of the indices of the variable nodes that are connected to the factor node $k$. Similarly, the BS rate $C_{0}$ can be approximated as a function of the subset $\mathbf{S}_{\rightarrow 0}$, where this subset is restricted to the variable nodes that are connected to the factor node 0 .

The interference constraint $(C 1)$ can be included in the optimization objective as a penalty function. If it is not fulfilled for D2D transmitter $k$, then this transmitter should not be active. The optimization function for D2D pair $k$ then becomes:

$$
\widehat{C}_{k}\left(\mathbf{S}_{\rightarrow k}\right)= \begin{cases}C_{k}\left(\mathbf{S}_{\rightarrow k}\right), & \text { if } I_{k, 0} \leq N_{r} I_{\max } \\ -\infty, & \text { otherwise }\end{cases}
$$

Let us set:

$$
\left\{\begin{array}{l}
M_{k}\left(\mathbf{S}_{\rightarrow k}\right)=-\widehat{C}_{k}\left(\mathbf{S}_{\rightarrow k}\right), \quad \forall k \in\{1, \ldots, K\} \\
M_{0}\left(\mathbf{S}_{\rightarrow 0}\right)=-C_{0}\left(\mathbf{S}_{\rightarrow 0}\right)
\end{array}\right.
$$

The optimization problem given in (16) can finally be written as follows:

$$
\min _{\mathbf{S} \in \mathcal{S}^{K}} M_{0}\left(\mathbf{S}_{\rightarrow 0}\right)+\sum_{k=1}^{K} M_{k}\left(\mathbf{S}_{\rightarrow k}\right)
$$

Problem (19) can be solved in a distributed way by using the min-sum message-passing algorithm on the factor graph which will be presented in Section III-B.

\section{B. Message-Passing Algorithm for resource allocation}

Before describing the min-sum message-passing algorithm, we first set some notations. Let $\mathcal{A}_{k}$ be the set of indices of variable nodes that are connected with factor node $k$ and $\mathcal{A}_{k} \backslash\{j\}$ the set of indices in $\mathcal{A}_{k}$ except index $j$. Finally, $\mathbf{s}_{\mathcal{A}_{k}}$ is the subvector from $\mathbf{s}$ restricted to the indices in $\mathcal{A}_{k}$.

The message-passing algorithm iteratively exchanges messages between factor nodes and variable nodes connected in the factor-graph, and vice-versa. Let $\mu_{M_{j} \rightarrow s_{k}}^{m}$ denote the message passed from factor node $M_{j}$ to variable node $s_{k}$, and $\mu_{s_{k} \rightarrow M_{j}}^{m}$ denote the message passed from variable node $s_{k}$ to factor node $M_{j}$, both at iteration $m$. The different steps of the min-sum MPA are given in Alg. 1.

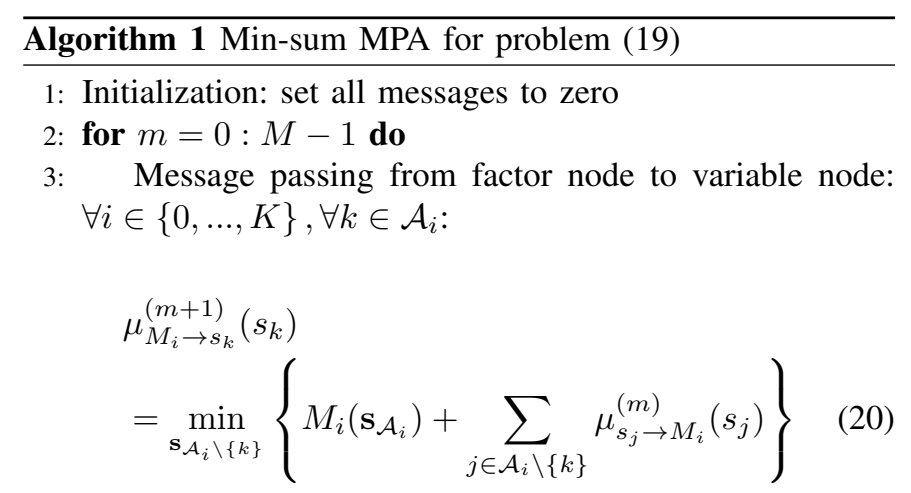

4: Message passing from variable node to factor node: $\forall k \in\{1, \ldots, K\}$ and $\forall i \in\{0, \ldots, K\}$ such that $k \in \mathcal{A}_{i}$ :

$$
\mu_{s_{k} \rightarrow M_{i}}^{(m+1)}\left(s_{k}\right)=\sum_{j \in \mathcal{A}_{k} \backslash\{i\}} \mu_{M_{j} \rightarrow s_{k}}^{(m+1)}\left(s_{k}\right)
$$

\section{5: end for}

6: Computation of the final parameters' values $s_{k}^{*}$ :

$$
s_{k}^{*}=\arg \min _{s_{k}}\left\{\sum_{j \in \mathcal{A}_{k}} \mu_{M_{j} \rightarrow s_{k}}^{(M)}\left(s_{k}\right)\right\}
$$

\section{Simulation Results}

We consider a $\mathrm{BS}$ in the cell with radius $R=0.5 \mathrm{~km}$. The noise is modeled as additive white Gaussian noise (AWGN) with power spectrum density $N_{0}=-174 \mathrm{dBm} / \mathrm{Hz}$. One active cellular user is selected among $K_{c}=25$ users. Therefore, one cellular user is allocated to a RB to establish transmission with target SINR value of $\mathrm{SINR}_{c}=20 \mathrm{~dB}$. D2D transmitters are in cell edge region defined from $R / 2$ to $R$. The maximum transmit power is fixed to $P_{\max }=11 \mathrm{dBm}$ at one $\mathrm{RB}$ for both $\mathrm{D} 2 \mathrm{D}$ transmitters and the cellular user. The bandwidth of one RB is defined as $B_{c}=180 \mathrm{kHz}$. The channel is modeled by using Rayleigh fading and shadowing follows log-normal distribution. The path loss and shadowing standard deviation both depend on whether the receiver is BS or a device. For the case that the receiver is the BS, the path loss model is $L=128.1+37.6 \log _{10}(d)$ where $d$ (in $\mathrm{km}$ ) is the distance between the transmitter and receiver and the standard deviation 
is equal to $9 \mathrm{~dB}$. For the case that the receiver is a device, the path loss model is $L=140+36.8 \log _{10}(d)$ where $d$ (in $\mathrm{km}$ ) is the distance between the transmitter and receiver and the standard deviation is equal to $4 \mathrm{~dB}$.

The connectivity constraint is determined as $I_{t h}=$ $-121 \mathrm{dBm}$ which is equal to $B_{c} N_{0}$. This threshold value causes relatively high interference between the nodes. The total estimated interference level at each D2D receiver and the BS are determined using Eq. (15), which correspond to the worst case in which all D2D pairs are actively communicating per RB.

In Fig 1, we demonstrate the effect of the maximum allowed interference per D2D pair at the BS, $I_{\max }$ on the system performance. Since the number of multiplexed D2D pairs directly depends on the interference level at the BS, for higher threshold values, the sum data rate is increased in the expense of degradation on the cellular data rate as shown in Fig 2. Then, we determine the maximum allowed interference per D2D pair at the BS as $I_{\max }=-121 \mathrm{dBm}$ that is also equal to connectivity constraint, $I_{t h}$, in order to maximize sum data rate while providing the cellular user the required data rate for a given $\operatorname{SINR}_{c}$.

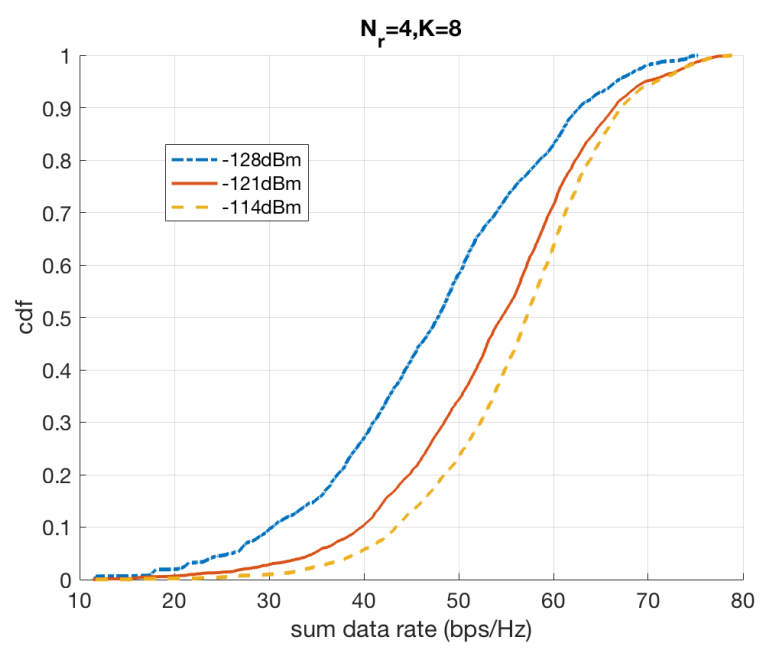

Figure 1: The effect of $I_{\max }$ values on the sum data rate.

In Fig 3, we demonstrate the effect of $T$ which corresponds to the different SINR requirements for the D2D pairs. The case of $T=3$ has $\operatorname{SINR}^{0}=0, \mathrm{SINR}^{1}=10 \mathrm{~dB}$ and $\mathrm{SINR}^{2}=20 \mathrm{~dB}$. The case of $T=2$ indicates D2D pair is not active with $\mathrm{SINR}^{0}=0$ and it is active with $\mathrm{SINR}^{1}=20 \mathrm{~dB}$. Under the same transmit power per node as shown in Fig 4, the sum data rate is increased significantly by assigning different SINR values for D2D pairs instead of completely shutting them. Then, the case of $T=3$ is kept for the rest of the simulation results.

The sum data rate performances of different resource allocation algorithms are shown in Fig. 5. The proposed algorithm based on MPA gives the same performance as exhaustive search with much less complexity. In Fig 6, it is illustrated that the transmit power per node is also the same for both

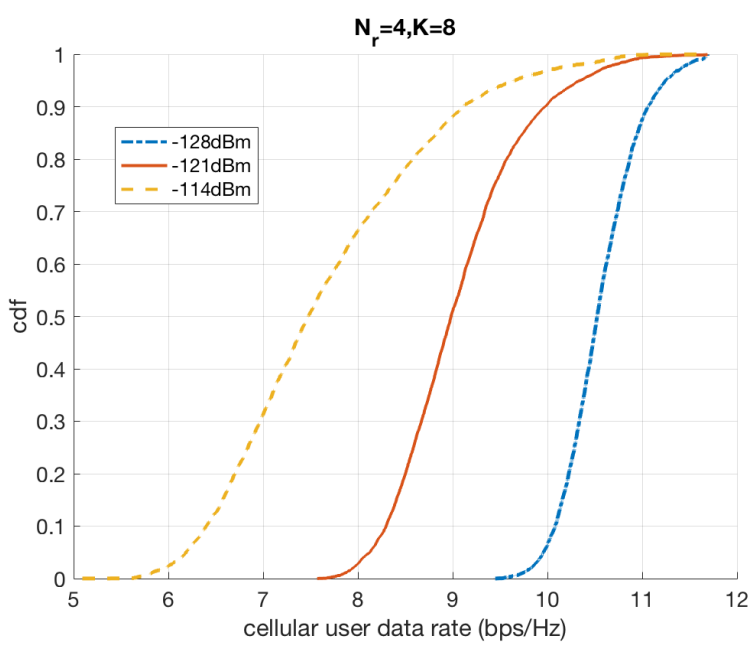

Figure 2: The effect of $I_{\max }$ values on the cellular user data rate.

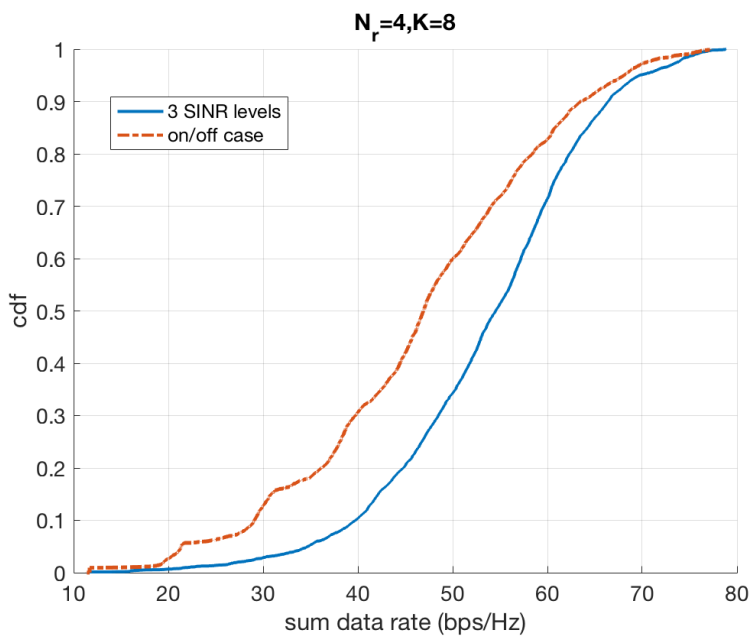

Figure 3: Comparison on sum data rate at different SINR requirements for D2D pairs

algorithms. Besides, the proposed algorithm outperforms the random allocation based resource allocation considering the sum data rate.

The average sum data rate is shown in Fig. 7 which illustrates that the average sum data rate is increased with the number of antennas and the number of $\mathrm{D} 2 \mathrm{D}$ pairs.

\section{CONClusion}

In this paper, we have examined the resource allocation problem in underlaying D2D multi-antenna cellular communications. In order to address for the sum data rate maximization of D2D communication without decreasing cellular data rate, we present MPA based resource allocation algorithm. First, we have constructed the factor graph of the D2D pairs based on the interference level among D2D pairs and at the BS. We have implemented the MPA iteratively and we have guaranteed nearly the best possible D2D pairs without causing a high level 


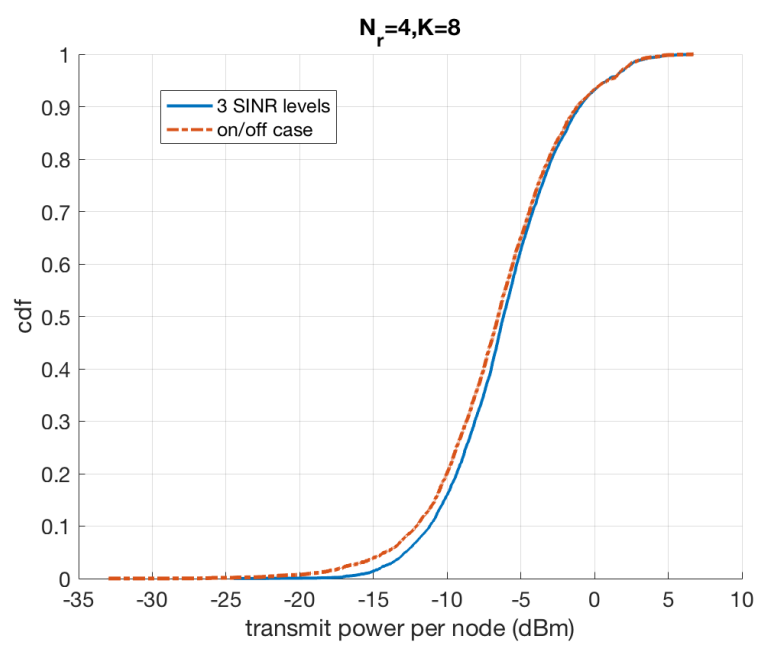

Figure 4: Comparison on transmit power per node at different SINR requirements for $\mathrm{D} 2 \mathrm{D}$ pairs

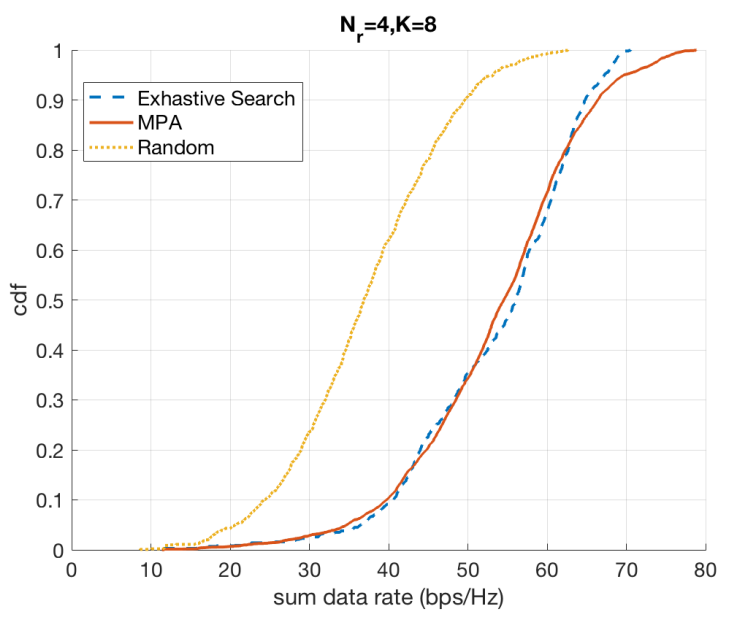

Figure 5: Comparison on sum data rate for different algorithms

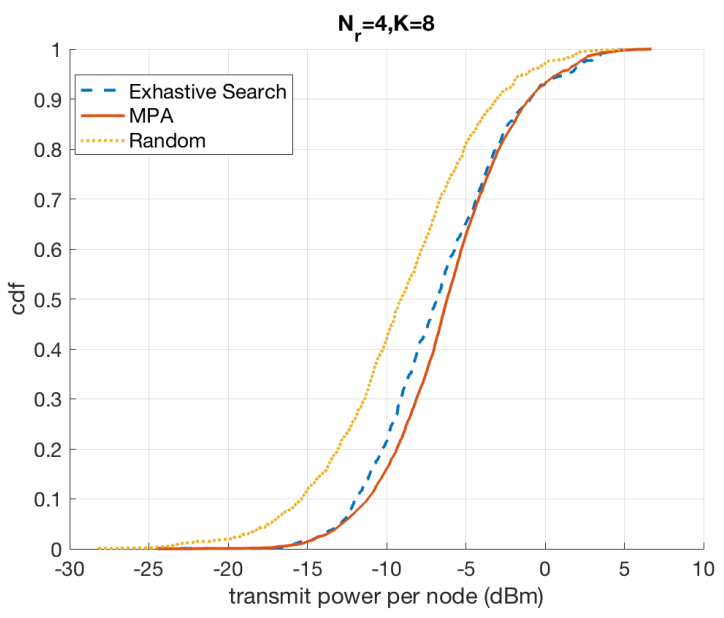

Figure 6: Comparison on transmit power per node for different algorithms

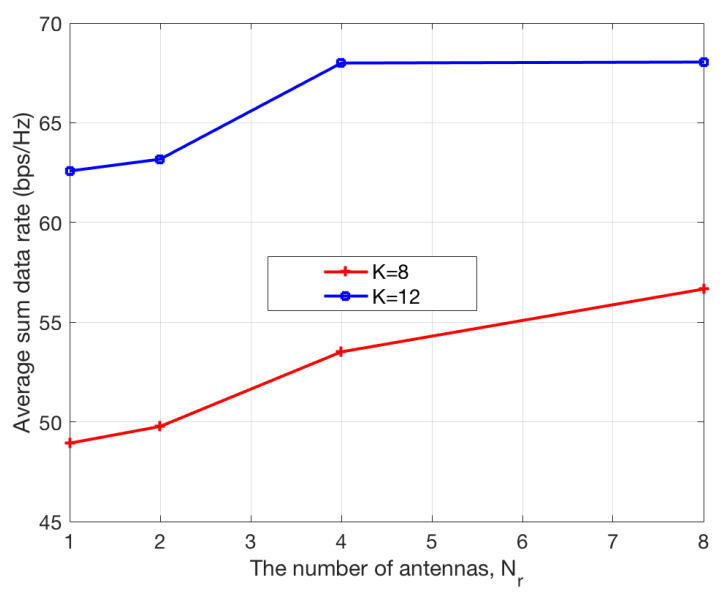

Figure 7: Comparison on average sum data rate for different D2D pairs and antennas at the BS

of interference at the BS. We have showed that the selection of the interference levels are very critical to maximize the sum data rate. As a future work, we will extend this scenario to the case of orthogonal frequency division multiple access (OFDMA) based cellular networks by considering multiple cellular users and multiple RBs.

\section{ACKNOWLEDGEMENT}

This work was partially funded through French National Research Agency (ANR) project ACCENT5 with grant agreement code: ANR-14-CE28-0026-02.

\section{REFERENCES}

[1] B. Ozbek, M. Pischella, and D. Le Ruyet, "Dynamic shared spectrum allocation for underlaying device-to-device communications," IEEE Wireless Communications, vol. 24, no. 5, pp. 88-93, October 2017.

[2] Xuejia Cai, Jun Zheng, and Yuan Zhang, "A graph-coloring based resource allocation algorithm for d2d communication in cellular networks," in Communications (ICC), 2015 IEEE International Conference on. IEEE, 2015, pp. 5429-5434.

[3] Tuong Duc Hoang, Long Bao Le, and Tho Le-Ngoc, "Resource allocation for $\mathrm{d} 2 \mathrm{~d}$ communication underlaid cellular networks using graph-based approach," IEEE Transactions on Wireless Communications, vol. 15, no. 10, pp. 7099-7113, 2016.

[4] F. Kschischang, B. J. Frey, and H. A. Loeliger, "Factor graphs and the sum-product algorithm," IEEE Transactions on information theory, vol. 47, no. 2, Feb. 2001.

[5] I. M. Guerreiro, D. Hui, and C. C. Cavalcante, "A distributed approach to precoder selection using factor graphs for wireless communication networks," EURASIP Journal on Applied Signal Processing, vol. 83, 2013.

[6] I. M. Guerreiro, C. C. Cavalcante, and D. Hui, "Adaptation with reducedsize message pass to precoder selection in multi-cell mimo systems," in 2016 IEEE Sensor Array and Multichannel Signal Processing Workshop (SAM), July 2016, pp. 1-4.

[7] A. Abrardo, M. Belleschi, P. Detti, and M. Moretti, "A min-sum approach for resource allocation in communication systems," in 2011 IEEE International Conference on Communications (ICC), June 2011, pp. 1-6.

[8] Y. Yu, M. Pischella, and D. Le Ruyet, "Distributed antenna selection with message passing algorithm for mimo d2d communications," in 2017 International Symposium on Wireless Communication Systems (ISWCS), Aug. 2017, pp. 1-6. 